\title{
Efficacy of fetal cardiac axis evaluation in the first trimester as a screening tool for congenital heart defect or aneuploidy
}

\author{
Youn-Joon Jung, MD*, Bo-Ra Lee, MD*, Gwang Jun Kim, MD, PhD \\ Department of Obstetrics and Gynecology, School of Medicine, Chung-Ang University, Seoul, Korea
}

\section{Objective}

To prove the efficacy of determining the abnormal fetal cardiac axis for screening congenital heart defects (CHDs) and predicting fetal aneuploidy at 11.0 to 13.6 weeks of pregnancy.

\section{Methods}

This retrospective study was performed at a single high-risk pregnancy center. The fetal cardiac axis was evaluated between 11.0 and 13.6 weeks of gestation in 142 fetuses. The cardiac axis in a 4-chamber view was measured as the angle between the line tracing the long axis of the heart and the line bisecting the thorax in the anteroposterior direction. A CHD was confirmed based on the second- to third-trimester fetal status or postnatal imaging. Aneuploidy was diagnosed using chorionic villus sampling, amniocentesis, or genetic testing after birth. Fisher's exact test was performed to assess the association between the fetal cardiac axis and the abnormal fetal status. A 2-way contingence table analysis was performed to confirm the efficacy of the fetal cardiac axis as a screening tool.

Results

Among the 142 fetuses, 10 had a CHD while 17 had aneuploidy. The abnormal fetal cardiac axis was significantly associated with CHDs $(P=0.013)$ and aneuploidy $(P=0.010)$. None of the fetuses with CHDs or aneuploidy had an isolated abnormal cardiac axis alone without other sonographic findings. The sensitivity of the fetal cardiac axis was $50.0 \%$ for CHDs and $41.2 \%$ for aneuploidy.

\section{Conclusion}

The fetal cardiac axis can be an additional helpful tool for prenatal screening of CHDs and aneuploidy in the first trimester.

Keywords: Fetal heart; Axis; Congenital heart defect; Aneuploidy; First trimester

\section{Introduction}

Congenital heart defects (CHDs) are the most common fetal congenital abnormality, independently or in association with other fetal anatomical defects. Prenatal identification of CHDs is important because these account for $30 \%$ to $50 \%$ of cases of infant death [1]. An optimal gestational age for screening for fetal cardiac anomalies is between 18 and 22 weeks of gestation [2]. However, early detection of CHDs in the first trimester is possible owing to the recent development of high-resolution ultrasonography [3]. In a 2006 systematic review of the first-trimester ultrasound examination for detecting major CHDs using transabdominal or trans-
Received: 2019.06.21. Revised: 2019.11.07. Accepted: 2019.11.12. Corresponding author: Gwang-Jun Kim, MD, PhD

Department of Obstetrics and Gynecology, School of Medicine, Chung-Ang University, 84 Heukseouk-ro, Dongjak-gu, Seoul 06974, Korea

E-mail: gjkim@cau.ac.kr

https://orcid.org/0000-0003-0232-0166

*These two authors contributed equally to this paper.

Articles published in Obstet Gynecol Sci are open-access, distributed under the terms of the Creative Commons Attribution Non-Commercial License (http://creativecommons. org/licenses/by-nc/3.0/) which permits unrestricted non-commercial use, distribution, and reproduction in any medium, provided the original work is properly cited.

Copyright $\odot 2020$ Korean Society of Obstetrics and Gynecology 


\section{Obstetrics \& Gynecology Science}

Youn-Joon Jung, et al. Fetal cardiac axis in first trimester

vaginal ultrasonography, the pooled sensitivity and specificity were $85 \%$ and $99 \%$, respectively [4]. Advantages of early detection of CHDs include earlier reassurance to highrisk women with normal sonographic findings, possibility of scheduling additional tests well before the limits for safe pregnancy termination, and provision of information for an appropriate place of birth and delivery methods depending on the severity of $\mathrm{CHD}$ [5].

Several methods have been suggested for screening of CHDs in early gestation, e.g., increased nuchal translucency (NT), abnormal blood flow in the ductus venosus (DV), and tricuspid regurgitation (TR) [6-11]. Based on previous studies, the abnormal cardiac axis has also been recognized as a screening marker for CHDs. Sinkovskaya et al. [12] reported that the efficacy of the cardiac axis measurement in the detection of major CHDs was significantly better than that of an NT scan, a DV blood flow scan, or tests for detecting TR, used alone or in combination.

CHDs are strongly associated with chromosomal anomalies and genetic syndromes. Identification of cardiac malformations on prenatal sonography substantially increases the risk for chromosomal abnormalities, with a frequency of aneuploidy reported to be as high as 22-32\% [13,14]. Increased NT, abnormal blood flow in DV, and TR, which are used in detection of CHDs, are also used for the screening of fetal aneuploidy in the first trimester [15-18].

We hypothesized that the abnormal fetal cardiac axis would be useful in the detection of fetal aneuploidy as well as fetal CHD during early gestation. The aim of this study was to investigate the effect of determining the abnormal cardiac axis for screening fetal CHD and predicting fetal aneuploidy at 11.0 to 13.6 weeks of pregnancy in the Korean population.

\section{Materials and methods}

From January 2015 to October 2018, 221 pregnant women in early gestation (11.0 and 14.6 weeks of gestation) who visited the Prenatal Diagnosis Clinic of the Chung-Ang University Hospital were enrolled in this study. One ultrasound image per woman was retrieved from the database. Images of 142 women were obtained after excluding those without precise 4-chamber views or neonatal information because of loss to follow-up. Clinical data and pregnancy outcomes were also collected from the database. Confirmation of CHD was based on the second- to third-trimester fetal status, postnatal imaging findings, or both. Aneuploidy of the fetuses was diagnosed using an invasive test, such as chorionic villus sampling and amniocentesis, during pregnancy or genetic testing after birth. A total of 52 chromosomal analyses were performed. In the first trimester, 47 fetuses were examined using chorionic villus sampling. Amniocentesis was performed on 4 fetuses in the second trimester. One fetus underwent genetic testing after birth. Invasive genetic tests were performed when the combined first trimester screening test or quad screening test resulted in a high risk, or when major fetal anomalies, including fetal hydrops, were observed on ultrasound. Chromosomal analyses were carried out using fluorescence in situ hybridization or classic karyotyping by long-term cell culture.

This study was undertaken by experts in the field of early fetal imaging. Target ultrasonography of the basic fetal anatomy including the 4-chamber view was performed routinely during early gestation. The 4-chamber view was obtained from an axial sonographic image with definite visualization of the cardiac chamber and 1 complete rib on each side of the fetal lateral chest wall. The cardiac axis was

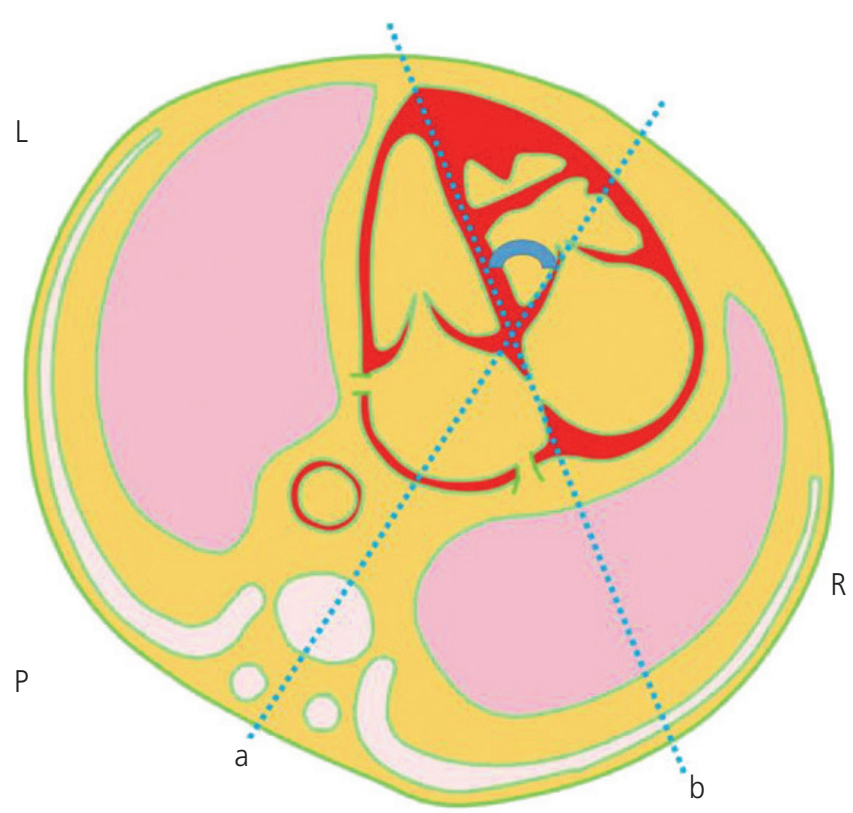

Fig. 1. A schematic diagram of the cardiac axis measurement. $a$, the line bisecting the thorax in the anteroposterior direction; $b$, the line tracing the long axis of the heart; $L$, left aspect of the fetus; $R$, right aspect of the fetus; $P$, posterior aspect of the fetus. 


\section{Obstetrics \& Gynecology Science}

Vol. 63, No. 3, 2020

measured as the angle between the line that traces the long axis of the heart and the line that bisects the thorax in the anteroposterior direction (Fig. 1). The normal fetal cardiac axis was defined as a $90 \%$ confidence interval $(\mathrm{Cl})$ of the cardiac axis in 121 fetuses without CHD or aneuploidy. Using the 1.65 standard deviation, range, and mean for fetuses without CHD or aneuploidy, the cardiac axis was considered as abnormal when the measured value was above the 95th percentile (left deviation) or below the 5th percentile (right deviation) (Fig. 2). The normal fetal cardiac axis range was calculated by rounding off the obtained value to the nearest whole number. Other first-trimester ultrasound findings, including the measurement of NT, blood flow in DV, presence of $T R$, and measurement of the nasal bone (NB) length, were also recorded. NT greater than 95th percentile or $3.0 \mathrm{~mm}$ was considered abnormal [19]. Reversal or absence of blood flow in DV was regarded as abnormal [16]. Absence of NB or an NB length less than the 2.5th percentile was considered abnormal [20]. Pregnancy-associated serum plasma protein A (PAPP-A) values were obtained from medical records to evaluate the screening performance for aneuploidy in combination with the abnormal fetal cardiac axis. Serum values of PAPP-A lower than 1.0 multiple of median were considered decreased.

The primary goal of this study was to investigate the association between the fetal cardiac axis in the first trimester and CHD and aneuploidy. The secondary goal of this study was to analyze the utility of the fetal cardiac axis measurement as a predictor of CHD and aneuploidy. Student's $t$-test and Fisher's exact test were performed to compare differences in clinical data between the study and control groups. The 2-way contingence table analysis was performed to confirm the efficacy of the fetal cardiac axis as screening tool. Bayes' theorem was used to compute the positive and negative predictive values for prevalence. Statistical analyses were performed using SPSS 25.0 (IBM Inc., Chicago, IL, USA). P<0.05 was considered statistically significant.

\section{Results}

Table 1 demonstrates the demographic and clinical characteristics of fetuses. Based on the fetal cardiac axis in the first trimester. In our collected data, 121 fetuses were not diagnosed with CHD or aneuploidy. In the group of normal fetuses, the cardiac axis ranged from $14.80^{\circ}$ to $75.70^{\circ}$ (mean $\left.47.15^{\circ} \pm 12.32^{\circ} ; 90 \% \mathrm{Cl}, 26.82-67.48\right)$. The normal fetal cardiac axis was defined as a value between $27^{\circ}$ and $67^{\circ}$. Based on the aforementioned definition of the normal cardiac axis, 24 fetuses had an abnormal cardiac axis. The incidence of the abnormal cardiac axis was significantly associated with increased NT, CHD, and aneuploidy. Decreased NB, abnormal Doppler finding on DV, and TR showed tendencies to occur more frequently in fetuses with an abnormal cardiac axis,
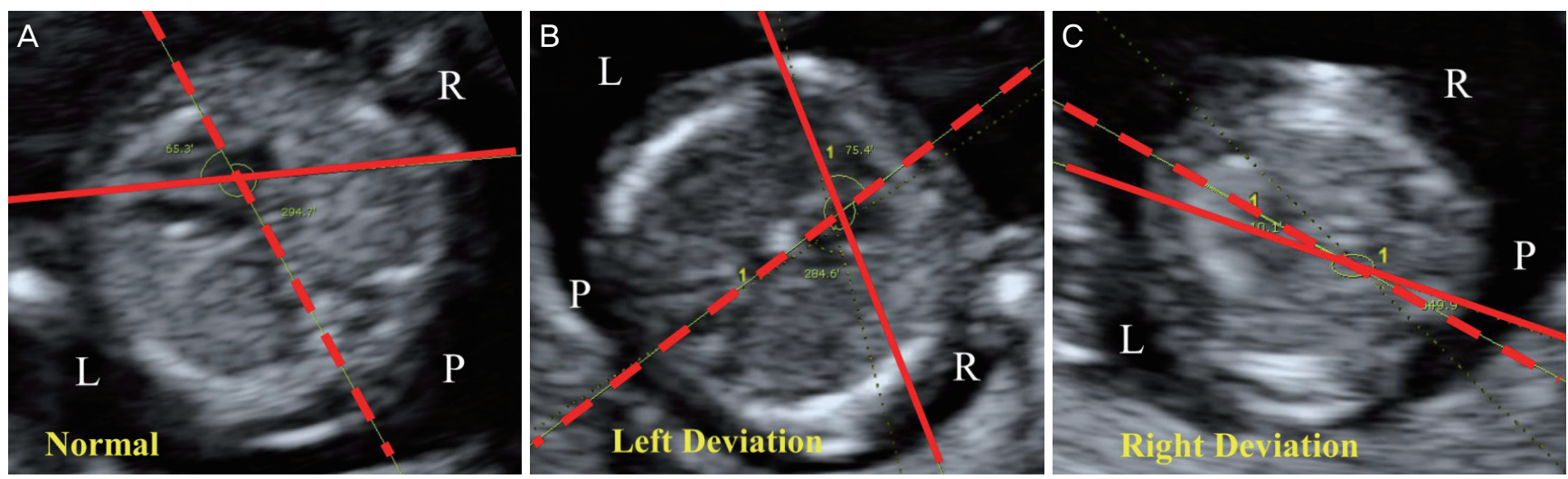

Fig. 2. Normal and abnormal fetal cardiac axes of fetuses in the first trimester observed using ultrasonography. Dotted lines are the lines bisecting the thorax in the anteroposterior direction. Full lines are the lines tracing the long axis of the heart. (A) Normal: $65.3^{\circ}$ at 11.0 weeks. The fetus was delivered through a cesarean section at 39.0 weeks of gestation. (B) Left deviation: $75.4^{\circ}$ at 12.4 weeks. Atrioventricular septal defect observed using fetal echocardiography. Trisomy 13: Fetal death in utero was diagnosed at 14.5 weeks of gestation. (C) Right deviation: $10.1^{\circ}$ at 12.2 weeks. Ventricular septal defect, aortic stenosis, mitral atresia, and left ventricular hypoplasia observed using fetal echocardiography. Fetal death in utero was diagnosed 13.4 weeks of gestation. $L$, left aspect of fetus; R, right aspect of fetus; $P$, posterior aspect of fetus. 


\section{Obstetrics \& Gynecology Science}

Youn-Joon Jung, et al. Fetal cardiac axis in first trimester

but without statistical significance. Aneuploidy without CHD was more common in fetuses with an abnormal cardiac axis $(12.50 \%)$ than in fetuses with a normal cardiac axis $(7.56 \%)$, but without statistical significance $(P=0.427)$. Among 142 registered fetuses, 10 fetuses were diagnosed with $C H D$ and 17 with aneuploidy. An abnormal cardiac axis was found in 5 of 10 fetuses with CHD and 7 of 17 fetuses with aneuploidy. Incidences of $\mathrm{CHD}$ and aneuploidy were associated with enlarged NT ( $P=0.002, C H D ; P<0.001$, aneuploidy), abnormal DV $(P<0.001, C H D ; P=0.001$, aneuploidy) and TR $(P<0.001$, $C H D ; P=0.003$, aneuploidy). Decreased NB was more common in fetuses with aneuploidy, but without statistical sig-

Table 1. Demographic and clinical characteristics of fetuses according to congenital heart defects (CHDs)

\begin{tabular}{lccc}
\hline Parameter & Normal cardiac axis & Abnormal cardiac axis & P-value \\
\hline No. of fetuses & 118 & 24 & - \\
Maternal age (yr) & $32.67 \pm 3.58(23-42)$ & $33.33 \pm 3.85(25-40)$ & 0.442 \\
CRL (mm) & $63.96 \pm 8.64$ & $62.81 \pm 9.90$ & 0.601 \\
Increased NT & $30(25.21)$ & $14(58.33)$ & 0.003 \\
Decreased NB & $16(13.45)$ & $7(29.17)$ & 0.054 \\
Abnormal DV & $12(2.52)$ & $3(12.50)$ & 0.709 \\
TR & $4(3.36)$ & $3(12.50)$ & 0.099 \\
Gestational age (wk) & $14(11.76)$ & $6(25.00)$ & - \\
$11.0-11.6$ & $68(57.14)$ & $10(41.67)$ & - \\
$12.0-12.6$ & $36(30.25)$ & $8(33.33)$ & - \\
$13.0-13.6$ & $5(4.20)$ & $5(20.83)$ & 0.013 \\
CHD & $10(8.40)$ & $7(29.17)$ & 0.010 \\
Aneuploidy & - & $15(62.50)$ & - \\
Left deviation & - & $9(37.50)$ & - \\
Right deviation & & & -
\end{tabular}

A normal cardiac axis was defined as that angled at $27-67^{\circ}$. Data are mean \pm standard deviation (range) or number (\%) unless specified otherwise. $P$-values were calculated by student's $t$-test or Fisher's exact test.

CRL, crown-rump length; NT, nuchal translucency; NB, nasal bone; DV, ductus venosus; TR, tricuspid regurgitation.

Table 2. Type of congenital heart defects (CHDs) and presence of abnormal cardiac axis in 10 fetuses

\begin{tabular}{|c|c|c|c|}
\hline CHD & $\begin{array}{l}\text { Abnormal } \\
\text { cardiac axis }\end{array}$ & Other sonographic abnormal findings & Aneuploidy \\
\hline AVSD, PA hypoplasia & Y & Cerebellar hypoplasia, Blake's pouch cyst & Trisomy 21 \\
\hline TOF & Y & - & $\mathrm{N}$ \\
\hline VSD & Y & Enlarged fetal bladder & Trisomy 21 \\
\hline MS, VSD, hypoplastic aortic arch & Y & - & Turner syndrome \\
\hline VSD & $\mathrm{N}$ & Fetal hydrops & $\mathrm{N}$ \\
\hline VSD & $\mathrm{N}$ & - & $\mathrm{N}$ \\
\hline VSD, aortic stenosis & $\mathrm{N}$ & Omphalocele, bilateral kidney agenesis & Trisomy 18 \\
\hline AVSD & $\mathrm{N}$ & Omphalocele, right dysplastic kidney & Trisomy 13 \\
\hline TS, VSD & $\mathrm{N}$ & - & $\mathrm{N}$ \\
\hline VSD, AS, mitral atresia, left ventricular hypoplasia & Y & Fetal hydrops & Turner syndrome \\
\hline
\end{tabular}

A normal cardiac axis was defined as that angled at $27-67^{\circ}$.

AS, aortic stenosis; AVSD, atrioventricular septal defect; MS, mitral stenosis; PA, pulmonary artery; TOF, tetralogy of Fallot; TS, tricuspid stenosis; VSD, ventricular septal defect. 


\title{
Obstetrics \& Gynecology Science
}

\author{
Vol. 63 , No. 3, 2020
}

nificance $(P=0.143)$. The incidence of CHD was significantly associated with aneuploidy $(P<0.001)$.

Overall, 10 fetuses with CHD were included in our study (Table 2). Five of them had an abnormal cardiac axis and 6 were diagnosed with aneuploidy. The most common CHD, regardless of the type, was ventricular septal defect (VSD). Table 3 demonstrates the frequency of the abnormal fetal cardiac axis for each type of aneuploidy. The most common manifestation of aneuploidy was Turner syndrome, and 4 fetuses with Turner syndrome showed an abnormal cardiac axis. Trisomy 21 was found in 3 patients, and all of whom had an abnormal cardiac axis.

Table 4 shows the efficacy of the fetal cardiac axis evaluation as a screening tool in the first trimester. With regard to $\mathrm{CHD}$, the sensitivity of the fetal cardiac axis evaluation was $50.0 \%(95 \% \mathrm{Cl}, 0.21-0.79)$, and the specificity was $85.6 \%$ ( $95 \% \mathrm{Cl}, 0.83-0.88)$. The negative predictive value of the fe-

Table 3. Frequency of abnormal fetal cardiac axis in each type of aneuploidy

\begin{tabular}{lcc}
\hline Aneuploidy & Number & $\begin{array}{c}\text { Abnormal cardiac axis } \\
\text { (number) }\end{array}$ \\
\hline Trisomy 12 & 1 & 0 \\
Trisomy 13 & 3 & 1 \\
Trisomy 18 & 3 & 2 \\
Trisomy 21 & 3 & 3 \\
Turner syndrome & 7 & 4 \\
\hline
\end{tabular}

A normal cardiac axis was defined as that angled at $27-67^{\circ}$. tal cardiac axis evaluation for CHD was 99.4\%. An abnormal cardiac axis in the first trimester increased the risk of CHD by approximately 6 times (odds ratio [OR], 5.95; $95 \% \mathrm{Cl}, 1.57-$ 22.52). With regard to aneuploidy, the sensitivity of the fetal cardiac axis evaluation was $41.2 \%(95 \% \mathrm{Cl}, 0.20-0.64)$, and the specificity was $86.4 \%(95 \% \mathrm{Cl}, 0.84-0.90)$. The negative predictive value of the fetal cardiac axis evaluation for aneuploidy was $99.9 \%$. An abnormal cardiac axis in the first trimester increased the risk of aneuploidy by approximately 4.5 times (OR, 4.45, 95\% Cl, 1.49-13.27).

Table 5 shows the efficacy of other variables alone and in combination with the fetal cardiac axis measurement as a screening tool in the first trimester. The sensitivity of NT in screening of CHD and aneuploidy were higher than that of the fetal cardiac axis. DV also had a higher sensitivity than the fetal cardiac axis in CHD and aneuploidy.

Table 4. The performance of fetal cardiac axis measurement as a screening tool in the first trimester

\begin{tabular}{lcc}
\hline Parameter & $\begin{array}{c}\text { Congenital heart } \\
\text { defects }\end{array}$ & Aneuploidy \\
\hline Sensitivity (\%) & $50.0(0.21-0.79)$ & $41.2(0.20-0.64)$ \\
$\begin{array}{l}\text { Specificity (\%) } \\
\begin{array}{l}\text { Positive predictive } \\
\text { value (\%) }\end{array}\end{array}$ & $85.6(0.83-0.88)$ & $86.4(0.84-0.90)$ \\
$\begin{array}{l}\text { Negative predictive } \\
\text { value (\%) }\end{array}$ & 9.8 & 0.4 \\
Odd ratio & 99.4 & 99.9 \\
\hline
\end{tabular}

Parentheses denote the $95 \%$ confidence interval.

Table 5. The performance of each of several independent variables and in combination with fetal cardiac axis measurement as a screening tool in the first trimester

\begin{tabular}{|c|c|c|c|c|c|c|}
\hline \multirow{2}{*}{ Parameter } & \multicolumn{2}{|c|}{ Sensitivity (\%) } & \multicolumn{2}{|c|}{ Specificity (\%) } & \multicolumn{2}{|c|}{$P$-value } \\
\hline & CHD & Aneuploidy & CHD & Aneuploidy & CHD & Aneuploidy \\
\hline NT & $80.0(0.45-0.96)$ & $82.4(0.58-0.95)$ & $72.3(0.70-0.74)$ & $75.6(0.72-0.77)$ & 0.008 & $<0.001$ \\
\hline DV & $66.7(0.30-0.90)$ & $42.7(0.20-0.66)$ & $93.0(0.91-0.95)$ & $92.7(0.90-0.95)$ & $<0.001$ & 0.001 \\
\hline $\mathrm{TR}$ & $40.0(0.15-0.61)$ & $25.0(0.09-0.38)$ & $97.7(0.96-0.99)$ & $97.6(0.96-0.99)$ & $<0.001$ & 0.003 \\
\hline Fetal cardiac axis and NT & $55.6(0.24-0.83)$ & $38.9(0.20-0.57)$ & $93.2(0.91-0.95)$ & $94.4(0.92-0.97)$ & $<0.001$ & $<0.001$ \\
\hline Fetal cardiac axis and DV & $33.3(0.11-0.33)$ & $16.7(0.05-0.17)$ & $100(0.99-1.00)$ & $100(0.98-1.00)$ & $<0.001$ & 0.002 \\
\hline Fetal cardiac axis and TR & $22.2(0.04-0.33)$ & $16.7(0.05-0.17)$ & $99.3(0.98-1.00)$ & $100(0.98-1.00)$ & 0.010 & 0.002 \\
\hline Fetal cardiac axis and PAPP-A $A^{\text {a) }}$ & - & - & - & - & - & 1.000 \\
\hline
\end{tabular}

Parentheses denote the $95 \%$ confidence interval. $P$-values were calculated by student's $t$-test or Fisher's exact test.

CHD, congenital heart defect; NT, nuchal translucency; DV, ductus venosus; TR, tricuspid regurgitation; PAPP-A, pregnancy-associated plasma protein A.

a) Fetal cardiac axis and PAPP-A was used only for screening for aneuploidy. 


\section{Obstetrics \& Gynecology Science}

Youn-Joon Jung, et al. Fetal cardiac axis in first trimester

\section{Discussion}

This study demonstrates that the fetal cardiac axis can be used for the prenatal sonographic screening of CHDs during early pregnancy in the Korean population. These results also suggest the possibility of the fetal cardiac axis being a screening tool for aneuploidy in the first trimester.

The possibility of the abnormal cardiac axis during early pregnancy being associated with CHD has been previously reported and has been consistent in our study [12]. In addition to this, consistent with previous studies, our study showed that increased NT, abnormal Doppler sonographic findings for DV blood flow, and TR were significantly more frequent in cases of CHD and aneuploidy [6-11,15-17].

CHDs were found in association with other anomalies or as part of an identified syndrome in $25-40 \%$ of cases [21]. In addition, approximately $30 \%$ of chromosomal abnormalities resulted in CHD [22]. In view of the association between CHD and aneuploidy, we speculated that an abnormal cardiac axis in the first trimester is correlated with aneuploidy. Our study showed a significant association between the fetal cardiac axis and aneuploidy.

Fetuses with aneuploidy but without CHD were not significantly associated with an abnormal fetal cardiac axis in the first trimester, although a tendency was observed. Since some fetuses with aneuploidy but without CHD had an abnormal cardiac axis, large scale studies are required to confirm this association.

NT is an important screening tool for CHDs and aneuploidy in the first trimester $[6,19,23]$. NT has a sensitivity of $56.0 \%$ and a negative predictive value of $99.9 \%$ for the prenatal diagnosis of CHDs in the first trimester [6]. The sensitivity of NT for aneuploidy has been reported to be $64-70 \%$ in the first trimester [24]. The NT scan was used as the first-trimester screening test for aneuploidy with serum free $\beta$ - or total human chorionic gonadotropin and PAPP-A analyte levels, and its detection rate varied from $82 \%$ to $87 \%$, depending on the gestational age [23]. As per our results, the fetal cardiac axis showed $50.0 \%$ sensitivity and $99.4 \%$ negative predictive value for $\mathrm{CHD}$ and $41.2 \%$ sensitivity and $99.9 \%$ negative predictive value for aneuploidy in 11.0 to 13.6 weeks of pregnancy. The fetal cardiac axis assessment in the first trimester may have value as a screening tool comparable to NT scanning.

However, our results showed that NT had a much higher sensitivity for screening CHD or aneuploidy than the abnormal cardiac axis. None of the fetuses with CHD or aneuploidy had an isolated abnormal cardiac axis alone without other sonographic findings. The abnormal cardiac axis alone in the first trimester seems insufficient to screen fetal CHD or aneuploidy. When the fetal cardiac axis was used for screening of CHD or aneuploidy with other sonographic findings, the specificity of the screening increased. Therefore, screening CHD or aneuploidy with the fetal cardiac axis assessment should be considered along with other sonographic findings.

Because sonographic assessment of a more detailed cardiac anatomy has been considered to be feasible at 11.0 to 13.6 weeks, we reviewed the data of mothers for corresponding weeks $[25,26]$. The measurement of NT is also appropriate during 11.0 to 13.6 weeks of the first trimester $[27,28]$. Further, the International Society of Ultrasound in Obstetrics and Gynecology recommends confirming the normal position of the heart on the left side of the chest (levocardia) simultaneously during NT measurement [28]. Thus, the fetal cardiac axis measurement is economical and convenient because it only requires the measurement of the axis in the existing surveillance procedure.

In previous studies, the 4-chamber view had the highest visualization rate at each gestational age and could be obtained in $85-100 \%$ of first-trimester ultrasound examinations $[29,30]$. Thus, the feasibility of cardiac axis measurement during early gestation using the transabdominal or transvaginal approach was established [31,32]. At our hospital, a sonographer with approximately 1 year of experience showed an almost 95\% chance of measuring the fetal cardiac axis in the first trimester. Specialized ultrasound skills are not required for the assessment of the fetal cardiac axis because the cardiac axis can be measured only in the 4-chamber view. The fetal cardiac axis measurement in the first trimester is valuable because screening is possible without the need for any hard training for measurement.

This study had several limitations. First, there may be a sampling bias. The Chung-Ang University Hospital is a tertiary hospital with a high proportion of high-risk pregnancy cases. Therefore, patients at this hospital may not represent the general population. Second, the cardiac axis was measured retrospectively. However, this was not likely to have a significant impact on the outcome as only pregnant women with a clearly visible interventricular septum in the 4-chamber view were included in the study. Third, the abnormal cardiac 


\title{
Obstetrics \& Gynecology Science
}

\author{
Vol. 63, No. 3, 2020
}

axis seemed to be less associated with VSD. In Tables 2 and 5 of 7 fetuses with VSD, which is the most common type of $\mathrm{CHD}$, had a normal fetal cardiac axis. This might be because 3 of them had an exceedingly small muscular VSD, which is thought to have a minor impact on the cardiac axis. Further analyses of the association between the abnormal cardiac axis and detailed types of VSD are warranted.

In conclusion, our study suggests that the fetal cardiac axis is valuable as a tool for the prediction of CHD in the first trimester. Further, we believe that the fetal cardiac axis may be used for the prenatal screening of aneuploidy in the first trimester. Since no fetuses with CHD or aneuploidy had an isolated abnormal cardiac axis alone, using the fetal cardiac axis alone for screening in the first trimester may not be appropriate. This conclusion requires confirmation through large-scale multicenter studies in the future.

\section{Conflict of interest}

No potential conflict of interest relevant to this article was reported.

\section{Ethical approval}

This retrospective study was approved by the Institutional Review Board of the Chung-Ang University Hospital (approval No. 1903-009-16255).

\section{Patient consent}

The patients provided written informed consent for the publication.

\section{References}

1. Heron M. Deaths: leading causes for 2014. Natl Vital Stat Report 2016;65:1-96.

2. Donofrio MT, Moon-Grady AJ, Hornberger LK, Copel JA, Sklansky MS, Abuhamad A, et al. Diagnosis and treatment of fetal cardiac disease: a scientific statement from the American Heart Association. Circulation
2014;129:2183-242.

3. Souka AP, Pilalis A, Kavalakis Y, Kosmas Y, Antsaklis P, Antsaklis A. Assessment of fetal anatomy at the 11-14week ultrasound examination. Ultrasound Obstet Gynecol 2004;24:730-4.

4. Rasiah SV, Publicover M, Ewer AK, Khan KS, Kilby MD, Zamora J. A systematic review of the accuracy of firsttrimester ultrasound examination for detecting major congenital heart disease. Ultrasound Obstet Gynecol 2006;28:110-6.

5. Kim GJ. Why we do the 1st trimester echocardiography. Ultrasound Med Biol 2017;43:S135.

6. Hyett J, Perdu M, Sharland G, Snijders R, Nicolaides $\mathrm{KH}$. Using fetal nuchal translucency to screen for major congenital cardiac defects at 10-14 weeks of gestation: population based cohort study. BMJ 1999;318:81-5.

7. Schwärzler P, Carvalho JS, Senat MV, Masroor T, Campbell S, Ville Y. Screening for fetal aneuploidies and fetal cardiac abnormalities by nuchal translucency thickness measurement at 10-14 weeks of gestation as part of routine antenatal care in an unselected population. $\mathrm{Br} J$ Obstet Gynaecol 1999;106:1029-34.

8. Timmerman E, Clur SA, Pajkrt E, Bilardo CM. Firsttrimester measurement of the ductus venosus pulsatility index and the prediction of congenital heart defects. Ultrasound Obstet Gynecol 2010;36:668-75.

9. Borrell A, Grande M, Bennasar M, Borobio V, Jimenez $J M$, Stergiotou I, et al. First-trimester detection of major cardiac defects with the use of ductus venosus blood flow. Ultrasound Obstet Gynecol 2013;42:51-7.

10. Pereira S, Ganapathy R, Syngelaki A, Maiz N, Nicolaides $\mathrm{KH}$. Contribution of fetal tricuspid regurgitation in firsttrimester screening for major cardiac defects. Obstet Gynecol 2011;117:1384-91.

11. Chelemen T, Syngelaki A, Maiz N, Allan L, Nicolaides KH. Contribution of ductus venosus Doppler in first-trimester screening for major cardiac defects. Fetal Diagn Ther 2011;29:127-34.

12. Sinkovskaya ES, Chaoui R, Karl K, Andreeva E, Zhuchenko L, Abuhamad AZ. Fetal cardiac axis and congenital heart defects in early gestation. Obstet Gynecol 2015; 125:453-60.

13. Crawford DC, Chita SK, Allan LD. Prenatal detection of congenital heart disease: factors affecting obstetric management and survival. Am J Obstet Gynecol 


\section{Obstetrics \& Gynecology Science}

Youn-Joon Jung, et al. Fetal cardiac axis in first trimester

1988;159:352-6.

14. Copel JA, Pilu G, Kleinman CS. Congenital heart disease and extracardiac anomalies: associations and indications for fetal echocardiography. Am J Obstet Gynecol 1986;154:1121-32.

15. Pandya PP, Kondylios A, Hilbert L, Snijders RJ, Nicolaides $\mathrm{KH}$. Chromosomal defects and outcome in 1015 fetuses with increased nuchal translucency. Ultrasound Obstet Gynecol 1995;5:15-9.

16. Matias A, Gomes C, Flack N, Montenegro N, Nicolaides $\mathrm{KH}$. Screening for chromosomal abnormalities at 10-14 weeks: the role of ductus venosus blood flow. Ultrasound Obstet Gynecol 1998;12:380-4.

17. Falcon O, Auer M, Gerovassili A, Spencer K, Nicolaides $\mathrm{KH}$. Screening for trisomy 21 by fetal tricuspid regurgitation, nuchal translucency and maternal serum free betahCG and PAPP-A at $11+0$ to $13+6$ weeks. Ultrasound Obstet Gynecol 2006;27:151-5.

18. Choi SJ. Clinical implications of nuchal translucency. Korean J Obstet Gynecol 2010;53:1-14.

19. Nicolaides KH, Azar G, Byrne D, Mansur C, Marks K. Fetal nuchal translucency: ultrasound screening for chromosomal defects in first trimester of pregnancy. BMJ 1992;304:867-9.

20. Cicero S, Longo D, Rembouskos G, Sacchini C, Nicolaides $\mathrm{KH}$. Absent nasal bone at 11-14 weeks of gestation and chromosomal defects. Ultrasound Obstet Gynecol 2003;22:31-5.

21. Fregin GF. Medical evaluation of the cardiovascular system. Vet Clin North Am Equine Pract 1992;8:329-46.

22. Pierpont ME, Basson CT, Benson DW Jr, Gelb BD, Giglia TM, Goldmuntz E, et al. Genetic basis for congenital heart defects: current knowledge: a scientific statement from the American Heart Association Congenital Cardiac Defects Committee, Council on Cardiovascular Disease in the Young: endorsed by the American Academy of Pediatrics. Circulation 2007;115:3015-38.

23. Dugoff L, Hobbins JC, Malone FD, Porter TF, Luthy D, Comstock $\mathrm{CH}$, et al. First-trimester maternal serum PAPP-A and free-beta subunit human chorionic gonado- tropin concentrations and nuchal translucency are associated with obstetric complications: a population-based screening study (the FASTER Trial). Am J Obstet Gynecol 2004;191:1446-51.

24. Malone FD, Ball RH, Nyberg DA, Comstock CH, Saade $G R$, Berkowitz RL, et al. First-trimester septated cystic hygroma: prevalence, natural history, and pediatric outcome. Obstet Gynecol 2005;106:288-94.

25. DeVore GR. First-trimester fetal echocardiography: is the future now? Ultrasound Obstet Gynecol 2002;20:6-8.

26. Yagel S, Cohen SM, Messing B. First and early second trimester fetal heart screening. Curr Opin Obstet Gynecol 2007;19:183-90.

27. McAuliffe FM, Fong KW, Toi A, Chitayat D, Keating S, Johnson JA. Ultrasound detection of fetal anomalies in conjunction with first-trimester nuchal translucency screening: a feasibility study. Am J Obstet Gynecol 2005; 193:1260-5.

28. Salomon LJ, Alfirevic Z, Bilardo CM, Chalouhi GE, Ghi T, Kagan KO, et al. ISUOG practice guidelines: performance of first-trimester fetal ultrasound scan. Ultrasound Obstet Gynecol 2013;41:102-13.

29. Haak MC, Twisk JW, Van Vugt JM. How successful is fetal echocardiographic examination in the first trimester of pregnancy? Ultrasound Obstet Gynecol 2002;20:913.

30. Smrcek JM, Berg C, Geipel A, Fimmers R, Diedrich K, Gembruch U. Early fetal echocardiography: heart biometry and visualization of cardiac structures between 10 and 15 weeks' gestation. J Ultrasound Med 2006;25:173-82.

31. Sinkovskaya E, Horton S, Berkley EM, Cooper JK, Indika $S$, Abuhamad A. Defining the fetal cardiac axis between $11+0$ and $14+6$ weeks of gestation: experience with 100 consecutive pregnancies. Ultrasound Obstet Gynecol 2010;36:676-81.

32. McBrien A, Howley L, Yamamoto $Y$, Hutchinson D, Hirose $A$, Sekar $P$, et al. Changes in fetal cardiac axis between 8 and 15 weeks' gestation. Ultrasound Obstet Gynecol 2013;42:653-8. 